\section{Atualizações em guias alimentares para crianças e adolescentes: uma revisão}

\section{Updating dietary guides for children and adolescents: a review}

Paula Martins Horta 1

Mariana Nunes Pascoal 2

Luana Caroline dos Santos 3

1-3 Grupo de Pesquisa em Intervenções em Nutrição. Departamento de Enfermagem Materno-Infantil e Saúde Pública. Universidade Federal de Minas Gerais. Av. Prof. Alfredo Balena, 190, sala 420. Bairro Santa Efigênia. Belo Horizonte, MG, Brasil. CEP: 30130-100. E-mail: hortapaula@yahoo.com.br

\title{
Resumo
}

Objetivos: apresentar uma revisão dos guias alimentares desenvolvidos para crianças e adolescentes.

Métodos: revisão sistemática conduzida nas bases de dados Medline e Lilacs e em sites institucionais. Incluíram-se documentos publicados entre 20002010.

Resultados: foram identificados 17 guias alimentares publicados no mundo, sendo dez na América Latina, três na América do Norte, dois na Europa e dois na Ásia e Oceania. Os guias se diferenciaram quanto à sua estrutura, podendo ser organizados em diretrizes nutricionais, representações gráficas ou em ambas. O conteúdo das diretrizes nutricionais, bem como o número de grupos alimentares e a representação gráfica utilizada nos manuais também são variáveis conforme cultura alimentar da população. Dezesseis guias enfocaram orientações sobre alimentos em detrimento dos nutrientes, sendo que o guia japonês está organizado em recomendações de consumo de preparações de alimentos, se configurando em um instrumento de mais fácil compreensão. Todos os guias alimentares identificados para crianças menores de dois anos $(n=5)$ foram elaborados na América Latina, além de terem sido detectados três manuais, cujas diretrizes se direcionam aos profissionais de saúde, denotando necessidade de capacitação e atualização dos mesmos.

Conclusões: o estudo apontou para a relevância do emprego dos guias alimentares na abordagem à criança e adolescente, fornecendo bases teóricas para profissionais e órgãos de saúde.

Palavras-chave Adolescente, Criança, Guias alimentares, Promoção da saúde 


\section{Introdução}

A prática de uma alimentação qualitativa e quantitativamente apropriada é essencial para o adequado crescimento e desenvolvimento dos seres humanos, sobretudo nos primeiros anos de vida. ${ }^{1}$ Desse modo, a promoção da alimentação saudável deve ser prioritariamente dirigida ao público infanto-juvenil, com destaque para a elaboração de guias alimentares. ${ }^{2}$

Os guias alimentares objetivam a promoção do bem-estar nutricional e a garantia do direito humano básico à alimentação através do fornecimento de orientações nutricionais sobre hábitos e práticas alimentares. ${ }^{3}$ Estas orientações podem ser transmitidas com auxílio de representações gráficas (pirâmide, arco-íris, roda, entre outros) ou por meio de diretrizes nutricionais. No primeiro caso, as representações simbolizam a alimentação dos indivíduos, sendo compostas por grupos alimentares. Para cada grupo alimentar tem-se uma recomendação de consumo em porções, relativa a como deve ser a participação dos alimentos deste grupo na alimentação diária do indivíduo. 4

As diretrizes nutricionais, por sua vez, fornecem informações qualitativas sobre os alimentos e orientam sobre os pressupostos de uma alimentação saudável utilizando linguagem coloquial e de fácil compreensão. Recomenda-se o emprego de mensagens curtas, claras e em número reduzido (entre seis e oito), que sejam positivas, não-prescritivas, culturalmente aceitas e sustentáveis, a fim de que a população, agentes de serviços públicos, sobretudo saúde e educação, possam assimilar com facilidade os conceitos abordados. 3

Entre os princípios e características dos guias alimentares, destaca-se que devem ser dirigidos à população-alvo, respeitando-se os hábitos alimentares locais e problemas de saúde relacionados, e que devem ser elaborados de acordo com recomendações científicas relacionadas à qualidade global da dieta e não quanto aos seus componentes isolados. Por fim, os guias alimentares devem ser simples, práticos e disseminados efetivamente, de forma a serem amplamente utilizados por indivíduos e profissionais de diversas áreas. 5

Alguns exemplos do uso de guias alimentares por diversos profissionais se relacionam ao seu emprego em ambientes institucionais como escolas, creches, orfanatos ou serviços de saúde. Moore et al., ${ }^{6}$ por exemplo, conduziram estudo de três meses com estudantes de nove à 11 anos $(\mathrm{n}=126)$, cuja intervenção foi constituída por seis aulas interativas, baseadas em jogo adaptado do guia alimentar infantil dos Estados Unidos.7 Já nos serviços de saúde, têm- se a iniciativa do Ministério da Saúde do Brasil, que elaborou um documento direcionado especificamente aos profissionais de saúde sobre como incluir as diretrizes do guia alimentar para menores de dois anos na atenção básica à saúde, de forma a aumentar o emprego deste instrumento neste nível de cuidado. ${ }^{8}$

Considerando a importância da educação nutricional para as condições de saúde das populações, o presente estudo objetivou apresentar uma revisão da literatura sobre guias alimentares desenvolvidos para crianças e adolescentes. Espera-se, com isso, divulgar aos profissionais, sobretudo da área da saúde e da educação, acerca dos diferentes guias alimentares direcionados ao público infanto-juvenil, bem como apresentar aos órgãos de saúde a metodologia e conteúdo dos guias alimentares já desenvolvidos, possibilitando a elaboração ou atualização destes instrumentos.

\section{Métodos}

Trata-se de artigo de revisão sistemática utilizandose como referencial teórico as recomendações propostas por Moher et al. 9 As bases de dados consultadas foram Medline (National Library of Medicine) e Lilacs (Literatura Latino-americana e do Caribe em Ciências da Saúde), além de terem sido visitados sites de órgãos de saúde (www.colombianutrinet.org; www.inha.sld.cu; www.inn.gob.ve; www.minsa.gob.pe; www.minsal. cl; www.mspbs. gov.py; www.portal.salud.gob.mx; www.saude.gov. br, www.sup.org.uy).

A pesquisa conduzida no Medline buscou identificar os guias alimentares publicados em todo o mundo, utilizando-se o termo "Nutrition policy" em associação aos termos "Nutritional requirements" e "Nutritional sciences/education". Já a pesquisa realizada na base de dados Lilacs, por sua vez, foi conduzida a partir do descritor "guias alimentares" e sua tradução em espanhol ("guías alimentarias") e inglês ("food guide") e objetivou identificar os guias alimentares desenvolvidos na América Latina.

Os passos adotados para a seleção dos guias alimentares em ambas as bases de dados foram os seguintes: inicialmente buscou-se identificar os artigos publicados entre 2000 e 2010 , em idioma inglês, português ou espanhol, cujos resumos estivessem disponíveis para consulta. A partir disso, foi conduzida uma leitura dos resumos dos documentos, selecionando-se aqueles cuja temática central fosse os guias alimentares. Após esta seleção, prosseguiu-se com a leitura íntegra dos manuscritos, sendo incluídos na pesquisa aqueles que tratassem 
do desenvolvimento de guias alimentares para crianças e adolescentes. Excluíram-se os artigos de revisão e os relacionados aos guias alimentares ainda em desenvolvimento, de abrangência local/regional ou que não estivessem vinculados aos órgãos de saúde dos países.

Além disso, os sites dos órgãos de saúde dos países da América Latina foram visitados no período de agosto de 2009 a fevereiro de 2010 , de forma a complementar a pesquisa sobre os guias alimentares elaborados para a população infanto-juvenil nessa região. Foram selecionados os sites que possuíssem guias alimentares para crianças e adolescentes desenvolvidos entre 2000-2010, cujo acesso era livre ao público.

Por fim, ressalta-se que os guias alimentares selecionados para a presente revisão estão organizados em diretrizes nutricionais, representações gráficas ou em ambos e foram analisados segundo essas classificações.

\section{Resultados}

Foram identificados 17 guias alimentares publicados no mundo, sendo dez na América Latina, três na América do Norte, dois na Europa e dois na Ásia e Oceania. A Figura 1 apresenta o fluxograma adotado para a seleção dos guias alimentares para a presente revisão.

A busca pelo Medline realizada pela associação dos termos "Nutrition policy" e "Nutritional requirements" identificou 186 artigos, sendo 60 (32,3\%) excluídos da análise por não atenderem aos critérios de inclusão da pesquisa. Assim, foram avaliados 126 artigos, porém somente $12(9,5 \%)$ faziam menção aos guias alimentares. Considerando o objetivo do presente estudo, três trabalhos foram integrados à revisão, sendo os mesmos referentes aos guias alimentares propostos para a população infantil alemã, 10 canadense ${ }^{11}$ e norteamericana. ${ }^{12}$

Já a pesquisa conduzida pela associação dos termos "Nutrition policy" e "Nutritional sciences/education" identificou 149 artigos, com exclusão inicial de 73 (49,0\%) documentos. Dos 76 artigos restantes, 15 (19,7\%) faziam menção aos guias alimentares, sendo incluídos ao estudo os referentes aos guias alimentares propostos para a população infantil da Austrália e Nova Zelândia,13 Japão14 e Portugal. 15

Para os artigos da América Latina, a pesquisa conduzida na base de dados Lilacs identificou sete artigos, sendo que somente um (14,3\%) - Guia alimentar para a população infantil argentina16 atendeu aos critérios de inclusão à presente revisão.
Os demais guias alimentares propostos para crianças e adolescentes da América Latina incluídos no presente estudo foram obtidos através dos sites do Ministério da Saúde do Brasil,8,17 Chile,18,19 Colômbia, ${ }^{20,21}$ Cuba, ${ }^{22,23}$ Paraguai ${ }^{24}$ e da Sociedade Uruguaia de Pediatria. ${ }^{25}$

A Tabela 1 mostra uma síntese das principais características dos guias alimentares identificados. A seguir, estes manuais foram descritos detalhadamente, utilizando-se a subdivisão didática em guias alimentares organizados em diretrizes nutricionais; guias alimentares organizados em representações gráficas e; guias alimentares organizados em representações gráficas e em diretrizes nutricionais.

\section{Guias alimentares organizados em diretrizes} nutricionais

Do total de guias alimentares identificados $(n=17)$, seis $(35,3 \%)$ são organizados somente em diretrizes nutricionais.

Iniciando-se pela Argentina, um guia alimentar dirigido à população infantil menor de dois anos foi publicado em 2006, pelo Ministério da Saúde do país. 16 Este guia está organizado em nove diretrizes que oferecem às famílias recomendações e práticas para o atendimento das necessidades que assegurem o crescimento e desenvolvimento adequados da criança. Dentre essas diretrizes destacam-se o incentivo ao aleitamento materno exclusivo nos primeiros seis meses de vida e complementado até os dois anos; fornecimento dos mesmos alimentos da família à criança com mais de um ano e; atenção especial à higiene e segurança alimentar. 16

O Chile, por sua vez, possui dois guias alimentares relacionados à alimentação da criança e do adolescente, sendo ambos organizados em diretrizes nutricionais. O guia alimentar para crianças menores de dois $\operatorname{anos}^{18}$ foi elaborado em 2005 a partir de um documento do Ministério da Saúde chileno intitulado "Norma de alimentação da criança menor de dois anos". Profissionais de saúde, especialistas e acadêmicos de todo o país decidiram estender a cobertura do guia alimentar e neste mesmo ano desenvolveram o guia que abrange a criança maior de dois anos até os adolescentes. ${ }^{19}$

O guia alimentar para crianças chilenas menores de dois anos oferece recomendações de incentivo ao aleitamento materno direcionadas aos profissionais de saúde de hospitais, consultórios e jardins de infância, através dos "Dez Passos para o Sucesso do Aleitamento Materno". Algumas recomendações incluem: criação de uma política de funcionamento que privilegie o aleitamento materno precoce, 

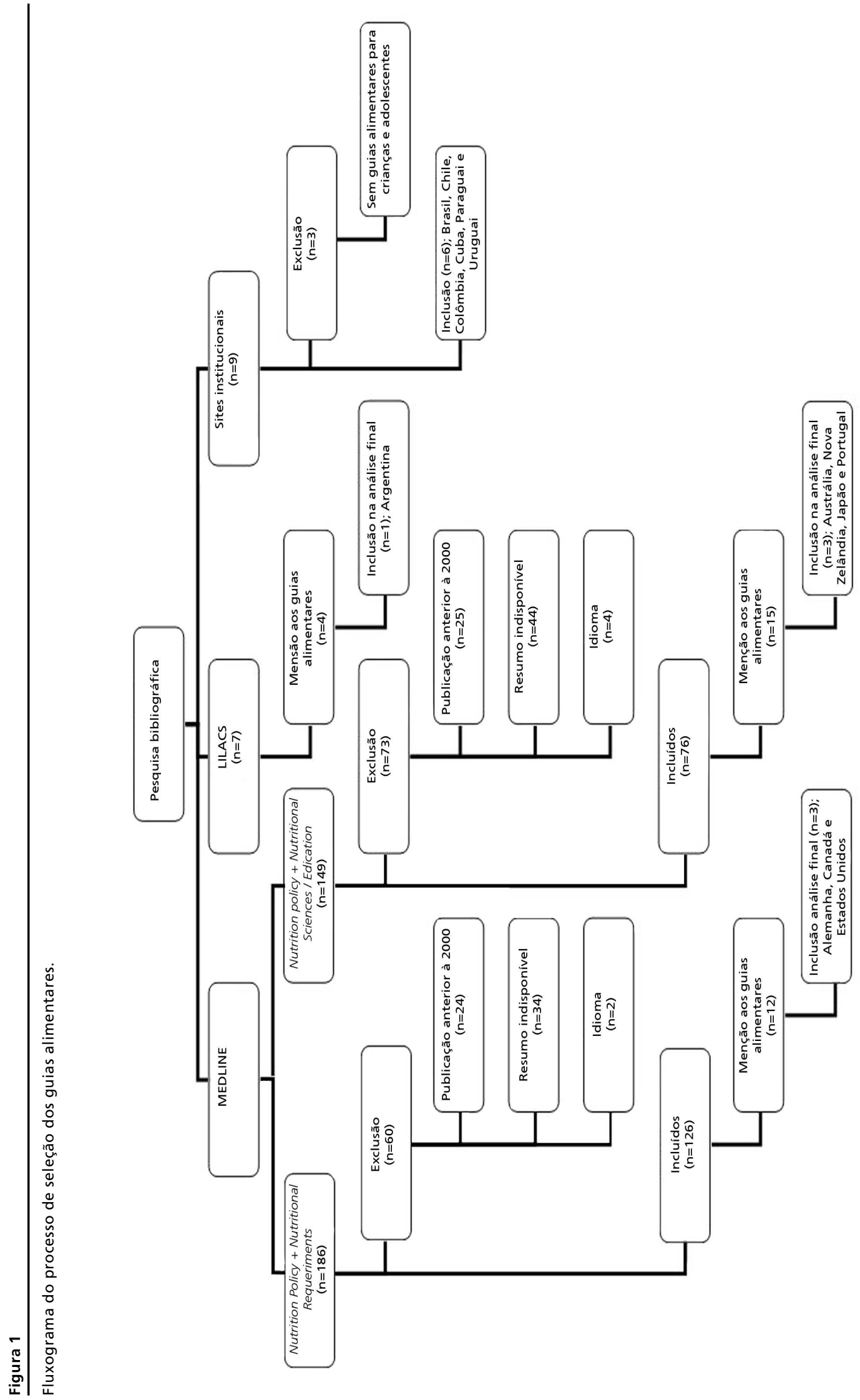
Síntese das características dos guias alimentares.

\begin{tabular}{|c|c|c|c|c|}
\hline País/ano & Público-alvo & $\begin{array}{c}\text { Representação } \\
\text { gráfica }\end{array}$ & $\begin{array}{c}\text { Grupos } \\
\text { alimentares }\end{array}$ & $\begin{array}{l}\text { Diretrizes } \\
\text { nutricionais }\end{array}$ \\
\hline Colômbia/2000 & Crianças maiores de dois anos & Locomotiva & Sete & Oito \\
\hline Brasil/2002* & Crianças maiores de dois anos & Pirâmide & Oito & Dez \\
\hline Austrália e Nova & Crianças e adolescentes & Pizza & Cinco & Quatro \\
\hline \multicolumn{5}{|l|}{ Zelândia/2003 } \\
\hline Cuba/2004 & Crianças maiores de cinco anos & Não possui & Sete & Nove \\
\hline Uruguai/2004 & Criança pré-escolar e escolar & Flor & Seis & Dezesseis \\
\hline Alemanha/2005 & Crianças maiores de um ano e adolescentes & Não possui & Não possui & Três \\
\hline Chile/2005 & Crianças menores de dois anos & Não possui & Não possui & Dez \\
\hline Chile/2005 & Crianças maiores de dois anos & Não possui & Não possui & Sete \\
\hline Colômbia/2005 & Crianças menores de dois anos & Locomotiva & Sete & Seis \\
\hline Estados Unidos/ 2005 & Crianças pré-escolares de dois à cinco anos & Pirâmide & Cinco & Não possui \\
\hline Estados Unidos/ 2005 & Crianças de seis à onze anos & Pirâmide & Cinco & Não possui \\
\hline Argentina/2006 & Crianças menores de dois anos & Não possui & Não possui & Nove \\
\hline Portuga,2006 & Crianças maiores de dois anos e adolescentes & Roda & Sete & Cinco \\
\hline Canadá/2007 & Crianças maiores de dois anos & Arco-íris & Quatro & Doze \\
\hline Japão/2007 & Crianças de seis à nove anos e adolescentes de 10 à 17 anos & Peão & Cinco & Não possui \\
\hline Cuba/2009 & Crianças menores de dois anos & Não possui & Sete & Seis \\
\hline Paraguai/2010 & Crianças menores de dois anos & Locomotiva & Não possui & Nove \\
\hline
\end{tabular}

*Revisado em 2010, com operacionalização dos “Dez Passos para uma alimentação saudável para as crianças brasileiras menores de dois anos".

através de treinamento de profissionais e orientações às mães; não oferecimento de outros alimentos, bebidas ou chupetas aos recém-nascidos, exceto em casos de indicação médica e; a comunicação com serviços de saúde e outras organizações da sociedade civil de forma a buscar apoio para suas ações. 18

Para as crianças chilenas acima de dois anos de idade, as recomendações contidas no guia alimentar são as mesmas para os demais grupos populacionais, contidas no guia educativo para uma vida saudável. ${ }^{19}$ Estas recomendações se configuram em um conjunto de sete diretrizes para uma alimentação saudável, sobretudo, para o consumo adequado de leite/derivados, frutas, hortaliças, pescados e água. O guia apóia ainda a prática de atividade física regular e o não tabagismo. 19

Cuba também possui guias alimentares direcionados às crianças e adolescentes, que são organizados em diretrizes nutricionais. Trata-se de um manual direcionado aos indivíduos com idade superior à cinco $\operatorname{anos}^{22}$ e outro aos menores de dois anos.23 Ambos os guias foram elaborados pelo Instituto de Nutrição e Higiene dos Alimentos do Ministério de Saúde Pública do país.22,23

O guia cubano para indivíduos com idade supe- rior à cinco anos foi elaborado em 2004 e está organizado em nove diretrizes nutricionais. A primeira recomendação é de incentivo à prática de uma alimentação variada, que deve incluir alimentos de sete grupos alimentares. As duas diretrizes seguintes se referem ao consumo diário de frutas e hortaliças. As outras recomendações se dirigem à ingestão de gorduras, carnes e pescados, sal, açúcar e realização do desjejum. Por fim, a nona diretriz apóia o controle do peso corporal. 22

Já o manual de Cuba para crianças menores de dois anos foi publicado em 2009 e está organizado em seis diretrizes que se dirigem aos profissionais de saúde e incentivam, dentre outros, o aleitamento materno exclusivo até os seis meses de vida, a amamentação como um ato de amor, a introdução de alimentação complementar a partir do primeiro semestre de vida, além da não adição de açúcar e sal aos alimentos naturais. O guia recomenda ainda o consumo diário de sete grupos alimentares, bem como disponibiliza opções para substituição de alimentos. 23

$\mathrm{Na}$ Europa, identificou-se o guia alimentar alemão para crianças e adolescentes entre um e 18 anos, elaborado em 2005, sob responsabilidade de 
seu Instituto de Pesquisa em Nutrição Infantil.10 A partir do estabelecimento de sete dietas diárias para cada grupo etário, houve organização dos alimentos em 11 subgrupos, contidos em três classes: a) bebidas e alimentos vegetais (bebidas, vegetais, frutas, pães/cereais, batata/massa); b) alimentos de origem animal (leite/derivados, carnes/salsichas, ovos, peixes) e; c) alimentos ricos em gordura e açúcar (alimentos tolerados - açúcar, doces, geléias, bolo, entre outros e óleos e gorduras). Para cada classe de alimentos foi definida uma proporção de consumo diário, somando-se três diretrizes nutricionais, que podem ser abordadas em atividades de educação nutricional, como por exemplo, pela associação com as cores do semáforo de trânsito. ${ }^{10}$

Guias alimentares organizados em representações gráficas

Do total de 17 guias alimentares identificados, apenas o guia alimentar japonês 14 é organizado somente em representação gráfica. Este manual foi criado em 2007 pelo Ministério da Saúde, do Trabalho e Bem-Estar e Ministério da Agricultura, Silvicultura e Pesca do país, e apresenta recomendações de consumo alimentar para sete grupos populacionais. Para as crianças, há referência para aquelas entre seis e nove anos e para os adolescentes entre 10 e 17 anos. 14

Este guia alimentar diferencia-se dos demais pelo fato de ser expresso em preparações - à base de cereais, de hortaliças, de carnes e peixes, leite/derivados e frutas - e não em alimentos isolados, oferecendo como vantagem a facilidade de compreensão das recomendações também pelos que desconhecem a forma de preparo dos alimentos. Além disso, a representação gráfica adotada para organização dos grupos de preparações foi o pião, devendo aquelas preparações posicionadas na parte superior serem consumidas em maiores quantidades ao longo do dia. Há um eixo central, que demonstra que o equilíbrio só é possível com a rotação, simbolizada pela prática regular de atividade física. ${ }^{14}$

Guias alimentares organizados em representações gráficas e em diretrizes nutricionais

Dos 17 guias alimentares identificados, 10 (58,8\%) são organizados em representações gráficas e em diretrizes nutricionais.

Considerando o guia alimentar brasileiro dirigido às crianças menores de dois anos, este foi elaborado com apoio da Organização Pan-
Americana de Saúde e constituiu uma das estratégias do Ministério da Saúde para a melhoria da saúde infantil. ${ }^{17} \mathrm{O}$ guia está organizado na forma de uma pirâmide, além de ser acrescido de dez diretrizes nutricionais. 17 Com relação à esta primeira, a pirâmide foi dividida em quatro níveis, selecionados pelo conteúdo de macro e micronutrientes. Nestes níveis estão organizados oito grupos alimentares, cuja participação na dieta encontra-se definida em número de porções. Junto à pirâmide encontra-se uma lista de substituição, na qual é definido o conteúdo de uma porção para 163 alimentos dos diferentes grupos alimentares. ${ }^{17}$

Já as diretrizes nutricionais propostas pelo guia brasileiro, estas se configuram nos "Dez passos para uma alimentação saudável para as crianças brasileiras menores de dois anos". Estas diretrizes foram recentemente operacionalizadas e direcionadas aos profissionais de saúde, objetivando esclarecer aos mesmos sobre como orientar a mãe ou responsável pela criança sobre a prática de alimentação infantil adequada. Esta nova versão foi acrescida ainda de informações e receitas sobre papas regionais, além de trazer conceitos atuais sobre alimentação infantil. 8

$\mathrm{Na}$ Colômbia, foram identificados dois guias alimentares, sendo um dirigido à população maior de dois anos 20 e outro aos indivíduos menores de dois anos. 21 O primeiro guia foi desenvolvido em 2000 pelo Instituto Colombiano de Bem-Estar Familiar (ICBF), um órgão do Ministério da Saúde, e se dirige aos indivíduos saudáveis de todas as faixas etárias, destacando-se as crianças e adolescentes de dois à 17 anos. 20 Com relação à sua estrutura, este guia possui uma representação gráfica e oito diretrizes nutricionais. A primeira se constitui em uma locomotiva, cuja cabine simboliza a família e os vagões os sete grupos alimentares. Para cada grupo alimentar foram definidas recomendações de consumo diário, variáveis conforme a faixa etária do indivíduo. ${ }^{20}$

Considerando as diretrizes nutricionais propostas neste guia, estas se somam em oito mensagens, relacionadas, por exemplo, ao incentivo da ingestão de frutas e hortaliças in natura, adequadamente higienizadas e sanitizadas, o controle do peso corporal e a prática de atividade física pelo menos três vezes por semana. O guia apóia ainda realização das refeições em família e a expressão de tolerância e solidariedade. 20

O guia elaborado para crianças colombianas menores de dois anos também foi elaborado pelo ICBF em 2005, possuindo, igualmente, a representação gráfica de uma locomotiva, além de seis dire- 
trizes nutricionais. ${ }^{21}$ A locomotiva também é composta por sete vagões representativos dos grupos alimentares, porém a cabine simboliza o aleitamento materno. Para este guia não são definidas quantidades específicas de porções dos alimentos que devem ser consumidas diariamente; entretanto, os vagões possuem tamanhos diferenciados, indicando como deve ser a proporção dos grupos alimentares na dieta. Já as seis diretrizes nutricionais presentes no guia são relativas à pratica da alimentação infantil adequada e incentivam o aleitamento materno exclusivo até os seis meses, introdução da alimentação complementar com itens dos sete grupos alimentares a partir desta fase, e a determinação de uma relação afetiva entre pais e filhos. ${ }^{21}$

Com relação ao Paraguai, identificou-se um guia alimentar, desenvolvido para crianças menores de dois anos, o qual foi revisado e atualizado pelo Instituto Nacional de Alimentação em 2010, órgão vinculado ao Ministério da Saúde. 24 A representação gráfica utilizada para este guia é uma locomotiva formada por uma cabine e três vagões. A cabine está representada pela alimentação nos primeiros seis meses de vida, correspondendo ao aleitamento materno exclusivo, enquanto os outros três vagões se referem aos alimentos que devem ser consumidos pelas crianças com seis a oito meses, nove a onze meses e um a dois anos, respectivamente. Além disso, este instrumento apresenta nove mensagens direcionadas aos pais ou responsáveis das crianças sobre como deve ser a alimentação adequada até os dois anos de vida. 24

O último guia alimentar organizado em uma representação gráfica e em diretrizes nutricionais identificado na América Latina se refere ao manual proposto pelo Uruguai à criança pré-escolar e escolar, elaborado em 2004 pela Sociedade de Pediatria do país. ${ }^{25}$ A representação gráfica utilizada é uma flor e cada pétala desta flor corresponde a um grupo alimentar. Ao todo são seis pétalas, de tamanhos diversos, indicando a contribuição diferenciada de cada um dos grupos para a alimentação diária da criança. Destaca-se que o miolo da flor é representado pela água, indicando que esta exerce um papel fundamental na promoção e manutenção da saúde. $25 \mathrm{O}$ documento possui ainda 16 diretrizes nutricionais para uma alimentação saudável, incluindo o consumo diário de frutas, hortaliças e leite; preferência aos óleos vegetais em detrimento das gorduras animais e; moderação no consumo de doces e alimentos industrializados. 25

Na América do Norte, foram identificados guias alimentares direcionados ao público infanto-juvenil no Canadá11 e Estados Unidos. 12 Este primeiro país possui um guia alimentar direcionado aos indivíduos com idade superior à dois anos e sua última versão foi publicada em 2007 pelo seu Ministério da Saúde. ${ }^{11}$ Para cada faixa etária foi elaborado um plano alimentar que abrange alimentos de quatro grupos: a) hortaliças e frutas; b) cereais e produtos derivados; c) leite e derivados; d) carne e derivados. ${ }^{11}$

Além disso, o guia canadense vêm acrescido de recomendações para escolha de melhores alimentos dentro de cada grupo alimentar e para diminuição do consumo de gorduras (recomendação de 30 a $45 \mathrm{~mL}$ por dia, preferencialmente sob a forma de óleos vegetais), açúcares e sal.11

Já nos Estados Unidos, o Departamento de Agricultura adaptou, em 2005, a pirâmide alimentar para a população geral para pré-escolares de dois a cinco anos e crianças de seis a onze anos. 12 Ambas as pirâmides são divididas em cinco faixas, cada uma representando um grupo alimentar: a) cereais, b) hortaliças, c) frutas, d) leite/derivados e e) carnes e feijões. Os grupos variam em tamanho, conforme a recomendação de consumo e possuem cores distintas. Estes guias são acrescidos de ilustrações de crianças praticando atividade física e de informações para moderação no consumo de óleo/gorduras e açúcares/doces. 12

$\mathrm{Na}$ Europa, o guia alimentar para a população portuguesa foi revisado e atualizado em 2006 pelo Ministério da Saúde do país.15 Este manual, apesar de ser direcionado à população geral, possui orientações de consumo alimentar para crianças e adolescentes. A representação gráfica utilizada é a roda de alimentos, que se divide em sete grupos alimentares, tendo a água posicionada no centro. Para cada grupo alimentar tem-se uma lista de substituição com a definição do conteúdo de uma porção para os diversos alimentos, além da recomendação de consumo diária. Destacam-se ainda cinco diretrizes que explicam como analisar a roda de alimentos e como fazer escolhas saudáveis na alimentação diária. 15

Já o guia alimentar australiano e neozelandês para crianças e adolescentes foi inicialmente proposto em 1995 e atualizado em 2003 pelo Conselho Nacional de Pesquisa Médica e Saúde. ${ }^{13}$ A representação gráfica utilizada é a mesma para a população adulta - pizza - dividida em cinco grupos de alimentos: a) cereais; b) hortaliças e leguminosas; c) frutas; d) leite/derivados; e) carne magra, peixes, aves e nozes. Para cada grupo alimentar tem-se a recomendação de consumo de porções que varia conforme a idade, sendo adotadas três classificações: 4 a 7 anos, 8 a 11 anos e 12 a 18 anos. Outros 
alimentos, como bolos, tortas, bebidas açucaradas, doces, etc, cujo consumo deve ser realizado ocasionalmente são representados externamente ao ícone.13

O guia é acrescido ainda de quatro diretrizes nutricionais referentes às melhores escolhas de alimentos dentro de cada grupo; ingestão alimentar e prática de atividade física regular, visando crescimento e desenvolvimento adequados; segurança alimentar e nutricional, além de incentivo ao aleitamento materno. 13

\section{Discussão}

Os resultados da pesquisa evidenciaram diferenças e semelhanças entre os diversos guias que merecem ser evidenciadas. Por exemplo, os guias alimentares propostos para os países da América Latina estão, em sua maioria, direcionados às crianças menores de dois anos, denotando a prevalente inadequação alimentar deste público nestes lugares. 16,17,18,21,23,24 Assim, muitos dos guias latino-americanos apresentam recomendações para o sucesso do aleitamento materno, que se assemelham bastante entre si. Entretanto, a diretrizes nutricionais contidas nos guias podem estar direcionadas aos profissionais de saúde, 17,18,23 à família16 ou não possuírem especificação do grupo populacional relacionado.11,13,15,20,21,25 Destaca-se ainda a inclusão da atenção à criança, criação de ambiente familiar de afeto e solidariedade nas diretrizes dos guias da Argetina, 16 Colômbia21 e Cuba23 como uma recomendação para o bom crescimento e desenvolvimento infantil.

Os guias alimentares diferenciam-se ainda com relação à sua organização, em diretrizes nutricionais, representações gráficas ou em ambos. O número de diretrizes nutricionais e de grupos alimentares, bem como o tipo de representação gráfica utilizada também é bastante variável entre os países, fato possivelmente explicado pelas diferenças de cultura alimentar. 26

Os achados do estudo denotaram ainda que os guias alimentares atuais têm enfocado preferencialmente a prevenção de doenças e agravos não transmissíveis, em detrimento às deficiências nutricionais. ${ }^{27}$ Tal mudança se deve à transição nutricional, pela qual passam muitos países, principalmente aqueles em desenvolvimento. Essa é definida como um fenômeno de inversão dos padrões de distribuição dos problemas nutricionais de uma dada população no tempo, ou seja, uma mudança na magnitude e no risco atribuível de agravos, sendo em geral, uma passagem da desnutrição para a obesidade. 28
Neste sentido, a prática de atividade física tem sido cada vez mais incentivada nos guias alimentares, objetivando a manutenção do peso saudável, seja na forma de orientações $13,19,20$ ou em ilustrações presentes nas representações gráficas. ${ }^{12,14}$

Adicionalmente, Barbosa et al. 26 apontam as mudanças ocorridas nas diretrizes nutricionais referentes à recomendação de consumo de lipídeos dos guias alimentares. As versões da década de 1980 não especificavam a quantidade do nutriente a ser ingerida, enquanto em 1990 já era recomendada a ingestão inferior à $30 \%$ do valor calórico total da dieta, sendo menos de $10 \%$ de gordura saturada. Atualmente, recomenda-se a redução do consumo também de gordura trans e colesterol, sendo que alguns guias já incorporam a recomendação para preferência de óleos vegetais, fontes de ácidos graxos mono e poliinsaturados, tal como o modelo proposto pelo Canadá. 10

Apesar desses avanços, muitos guias alimentares ainda se referem ao grupo das gorduras e dos açúcares de maneira vaga e pouco clara, por meio de expressões como: "uso moderado", "pequena quantidade", "quantidade razoável", dificultando sua interpretação. 26 Neste sentido, os guias propostos para a população brasileira 17 e canadense 10 se destacam por oferecerem uma recomendação de consumo de óleos e gorduras em porções e em volume $(\mathrm{mL})$ de alimentos, respectivamente.

Outra importante mudança que pode ser constatada nos guias alimentares com o passar dos anos, se refere à abordagem da alimentação como um todo, evitando-se o foco nos nutrientes. Essa mudança de concepção representa, na prática, a elaboração de instrumentos mais claros e fáceis de serem compreendidos pela população. Um guia desenvolvido puramente em base cientifica, cujas recomendações são expressas em termos de energia e nutrientes necessitam, para seu entendimento, de conhecimento cientifico especializado. 14

Entretanto, a própria abordagem do guia em alimentos apresenta limitações, uma vez que estes são ingeridos como preparação devendo ser classificados em mais de um grupo alimentar. Para isso se faz necessário desmembrar os ingredientes, tornando obrigatório o conhecimento da receita da preparação. ${ }^{14,26}$ Objetivando minimizar esse problema, o guia alimentar proposto para a população japonesa está organizado em grupos de preparações, esperando-se com isso maior compreensão das recomendações pelos indivíduos que desconhecem os ingredientes da preparação. Entretanto, para avaliar os reais benefícios desta maneira de organizar as 
recomendações, são necessários maiores estudos. 14

Quanto às populações abordadas nos guias alimentares, o que se observa é uma maior prevalência daqueles direcionados à população menor de dois anos, principalmente em países em desenvolvimento, contrastando com a população juvenil. Isso provavelmente se explica pelas características dessa faixa etária, que incluem necessidades nutricionais específicas para adequado crescimento e desenvolvimento, além dos frequentes problemas de saúde que se relacionam à alimentação. ${ }^{17}$ Adicionalmente, muito embora se verifique uma tendência à redução na prevalência da desnutrição energético-protéica nos países em desenvolvimento, os dados indicam que este quadro clínico vem se concentrando entre as crianças de seis a 18 meses de idade. 17

Além disso, constata-se também direcionamento das orientações nutricionais aos profissionais de saúde, principalmente referentes ao aleitamento materno. Estudo conduzido com 875 pediatras demon- strou que estes são menos propensos a acre-ditar que os benefícios do aleitamento materno superam as dificuldades e os inconvenientes e que poucos acreditam que a quase totalidade das mães são capazes de amamentar durante o período recomendado. 29 Associado a isso, verifica-se baixa duração de aleitamento materno exclusivo, constatando-se mediana nacional no Brasil de 54,11 (IC95\%: 50,30-57,73) dias, ${ }^{30}$ denotando necessidade de implementação de estratégias de incentivo à amamentação.

Em síntese, os guias alimentares representam um importante instrumento para atender os objetivos dos programas de educação nutricional, pois facilitam a aprendizagem e adaptação de uma conduta alimentar saudável garantindo o direito universal de cidadania. O presente estudo apresentou uma revisão sobre os guias alimentares propostos para a população infanto-juvenil, fornecendo bases teóricas para profissionais, sobretudo da área de saúde e educação, e órgãos de saúde para uma maior aplicação destes manuais nos serviços de saúde.

\section{Referências}

1. WHO (World Health Organization). UNICEF (The United Nations Children's Fund). Department of Child and Adolescent Health and Development. Planning guide for national implementation of the Global Strategy for Infant and Young Child Feeding. 2007. [Acesso em 20 ago 2009]. Disponível em: <www.who.int>

2. Anderson GH, Zlotkin SH. Developing and implementing food-based dietary guidance for fat in the diets of children. Am J Clin Nutr. 2000; 72: 1404-9.

3. Harris S, Black R, Harvey AG. Dietary guidelines: past experience and new approaches. Am J Diet Assoc. 2003; 103: 3-4.

4. Molina V. Guías Alimentarias en America Latina. Informe de la consulta técnica regional de las Guías Alimentarias. Ann Venez de Nutr. 2008; 21: 31-41.

5. WHO (World Health Organization). FAO (Food and Agriculture Organization of the United Nations). FAO/WHO Technical Consultation on National FoodBased Dietary Guidelines: Cairo, Egypt. 6-9 December, 2004. 2006. 80 p.

6. Moore JB, Pawloski LR, Goldberg P, Kyeung MO, Stoehr A, Baghi H. Childhood obesity study: a pilot study of the effect of the nutrition education program Color $\mathrm{My}$ Pyramid. J Sch Nurs. 2009; 25: 230-9.

7. USDA (United States Department of Agriculture). Center for Nutrition Policy and Promotion. MyPyramid. Steps to a Healthier You. 2005. [Acesso em 20 ago 2009]. Disponível em: <www.mypyramid.gov>

8. Brasil. Ministério da Saúde. Secretaria de Atenção à Saúde. Departamento de Atenção Básica. Dez passos para uma alimentação: guia alimentar para crianças menores de dois anos: um guia para o profissional da saúde na atenção básica; $2010.72 \mathrm{p}$
9. Moher D, Liberati A, Tetzlaff J, Altman DG, PRISMA Group. Preferred reporting items for systematic reviews and meta-analysis: the PRISMA statement. Ann Intern Med. 2009; 151: 264-9.

10. Kersting M, Alexy U, Clausen K. Using the concept of food-based dietary guidelines to develop an Optimized Mixed Diet (OMD) for German children and adolescents. J Pedriatr Gastroenterol Nutr. 2005; 40: 301-8.

11. Katamay SW, Esslinger KA, Vigneault M, Johnston JL, Junkins BA, Robbins LG, Sirois IV, Jones-Mclean EM, Kennedy AF, Bush MA, Brulé D, Martineau C. Eating well with Canada's Food Guide (2007): development of the food intake pattern. Nutr Rev. 2007; 65: 155-66.

12. Chiuve SE, Willett WC. The 2005 Food Guide Pyramid: an opportunity lost? Nat Clin Pract Cardiovasc Med. 2007; 4: 610-20.

13. Baghurst K. Dietary guidelines: the development process in Australia and New Zealand. J Am Diet Assoc. 2003; 103: 17-21.

14. Yoshiike N, Hayashi F, Takemi Y, Mizoguchi K, Seino F. A new food guide in Japan: The Japanese Food Guide Spinning Top. Nutr Rev. 2007, 65: 149-54.

15. Rodrigues SSP, Franchini B, Graça P, de Almeida MDV. A new food guide for the Portuguese population: development and technical considerations. J Nutr Educ Behav. 2006, 38 : 189-95.

16. Longo EM, Albaizeta D. Guías alimentarias para la población infantil. Diaeta. 2006; 24: 28-31.

17. Brasil. Ministério da Saúde. Secretaria de Política da Saúde. Organização Pan-Americana de Saúde. Guia Alimentar para crianças menores de dois anos; 2002. 152 p. 
18. Chile. Ministerio de Salud. Guía de alimentación del niño(a) meñor de 2 años. Guías de alimentación hasta la adolescencia; $2005.70 \mathrm{p}$

19. Chile. Ministerio de Salud. Guía educativa para una vida saudable. Guias alimentarias, actividad física y tabaco; 2005. 20 p.

20. Colombia. Ministerio de Salud. Guías Alimentarias para la población colombiana mayor de dos años: bases tecnicas; 2000.56 p.

21. Colombia. Ministerio de Salud. Guías Alimentarias para niños y niñas colombianos menores de 2 años; 2005 [Acesso em 20 ago 2009] Disponível em: <www.colombianutrinet.org>

22. Cuba. Ministerio de Salud Publica. Dirección Naciona Materno Infantil. Instituto de Nutrición e Higiene de los Alimentos. Una propuesta de vida saludable. Guías alimentarias para la población cubana mayor de dos años de edad; 2004. [Acesso em 20 ago 2009]. Disponível em: $<$ www.inha.sld.cu $>$

23. Cuba. Ministerio de Salud Publica. Dirección Nacional Materno Infantil. Instituto de Nutrición e Higiene de los Alimentos. Guías Alimentarias para niños y niñas cubanos hasta 2 años de edad - Documento Tecnico para los Equipos de Salud; 2009. 80 p.
24. Paraguay. Ministerio de Salud Publica y Bienestar Social. Instituto Nacional de Alimentación y Nutrición. Dirección General de Programas de Salud. Comitê Técnico Nacional de las Guías Alimentarias del Paraguay. Guías Alimentarias para niñas y niños menores de 2 años del Paraguay; 2010. $127 \mathrm{p}$.

25. SUP (Sociedade Uruguaya de Pediatría). Comité de Nutrición. Guías de alimentación del niño preescolar e escolar. Arch Pediatr Urug. 2004, 75: 159-63.

26. Barbosa RMS, Salles-Costa R, Soares EA. Guias alimentares para crianças: aspectos históricos e evolução. Rev Nutr. 2006; 19: 255-63.

27. Schneeman B. Evolution of dietary guidelines. J Am Diet Assoc. 2003; 103: 5-9.

28. Doak CM, Adair LS, Bentley M, Monteiro C, Popkin BM. The dual burden household and the nutrition transition paradox. Int J Obs. 2005, 29: 129-36.

29. Feldman-Winter LB, Schanler RJ, O'Connor KG, Lawrence RA. Pediatricians and the promotion and support of breastfeeding. Arch Pediatr Adolesc Med. 2008; 162: 1142-50.

30. Brasil. Ministério da Saúde. Secretaria de Atenção à Saúde. Departamento de Ações Programáticas e Estratégicas. II Pesquisa de prevalência de aleitamento materno nas capitais brasileiras e Distrito Federal; 2009. 108 p.

Recebido em 11 de janeiro de 2010

Versão final apresentada em 25 de fevereiro de 2011

Aprovado em 22 de março de 2011 